\title{
Charge Separation and Charge Carrier Mobility in Photocatalytic Metal-Organic Frameworks
}

Maria Fumanal*a,b, Andres Ortega-Guerrero ${ }^{a}$,Kevin Maik Jablonka, ${ }^{a}$ Berend Smit ${ }^{a}$, Ivano Tavernelli*b

\begin{abstract}
Metal-Organic Frameworks (MOFs) are highly versatile materials owing to their vast structural and chemical tunability. These hybrid inorganic-organic crystalline materials offer an ideal platform to incorporate lightharvesting and catalytic centers and thus, exhibit a great potential to be exploited in solar-driven photocatalytic processes such as $\mathrm{H}_{2}$ production and $\mathrm{CO}_{2}$ reduction. To be photocatalytically active, UV-visible optical absorption and appropriate band alignment with respect to the target redox potential is required. Despite fulfilling these criteria, the photocatalytic performance of MOFs is still limited by their ability to produce long-lived electron-hole pairs and long-range charge transport. In this work, we establish a cost-effective computational strategy to address these two descriptors in MOF structures and translate them into charge transfer numbers and effective mass values. We apply our approach to $15 \mathrm{MOF}$ structures from the literature that encompass the main strategies used in the design of efficient photocatalysts including different metals, ligands, and topologies. Our results capture the main characteristics previously reported for these MOFs and enable us to identify promising candidates. In the quest of novel photocatalytically active systems, high-throughput screening based on charge separation and charge mobility features is envisioned to be applied in large databases of both experimentally and in silico generated MOFs.
\end{abstract}

\section{Introduction}

Metal-Organic Frameworks (MOFs) are porous crystalline materials composed of metal clusters connected by organic linkers. ${ }^{1,2}$ Because of their modular nature, MOFs possess a unique synthetic versatility that has been exploited in a wide range of applications ${ }^{3}$ from gas storage and gas separation ${ }^{4}$ to catalysis ${ }^{5}$ and biomedical sensing. ${ }^{6}$ Recently, great interest has also emerged in the use of MOF to address solar-driven photocatalytic reactions including hydrogen production ${ }^{7}, \mathrm{CO}_{2}$ reduction ${ }^{8}$, pollutant degradation ${ }^{9}$ and organic transformations. ${ }^{10}$ The potential of MOFs as photocatalysts relies on the appropriate combination of visible-light-responsive organic ligands with oxidizing or reducing metal centers. ${ }^{11}$ To be photocatalytically active, a MOF material must fulfill two energetic requirements, namely, visible-light absorbance and appropriate band-edge alignment with respect to the desired redox partners. These two characteristics can be associated with two energy-based descriptors that are accessible through electronic structure calculations. In particular, the light-absorption capabilities can be associated with the optical gap of the MOF material ${ }^{12}$ and the band-edge energies can be efficiently estimated with respect to the vacuum potential evaluated at the center of the pore. ${ }^{13}$

While the capability of a MOF material to undergo photocatalysis is mainly determined by its visible-light absorbance and band-edge energies, its performance has been directly associated with the lifetime of the photogenerated charge carriers (electrons and holes), as well as with the charge-carriers mobility. ${ }^{14}$ The main strategy used to increase the lifetime of the photo-generated charge carriers is to promote spatial charge separation in the low-lying excited states. ${ }^{15}$ One possibility is to perform metal substitutions that result in low-lying unoccupied states localized in the metal nodes. ${ }^{16,17}$ These substitutions lead to low-lying ligand-to-metal charge-transfer (LMCT) states when the highest occupied levels are localized in the organic ligand (Figure 1A). It is also possible to stabilize lowlying metal-to-ligand charge-transfer (MLCT) states by combining easily oxidizable metals and reducible ligands (Figure 1B). ${ }^{18,19}$ In both cases, LMCT and MLCT, the photo-generated charges are spatially separated in either the ligand or the metal cluster of the MOF material in favor of long-lived lifetimes and low recombination rates. In contrast, low lying excitations towards ligand-centered (LC) and metal-centered (MC) states (Figure 1C and Figure 
1D, respectively) are associated with short and intense emission exploited in photo-luminescent applications. ${ }^{20,21}$ It is worth mentioning that light-induced ligand-to-ligand charge transfer (LLCT) (Figure 1E) and metal-to-metal charge transfer (MMCT) (Figure 1F) has been reported in $\mathrm{MOFs}^{22,23}$ and could also be exploited to enlarge the electron-hole lifetimes.

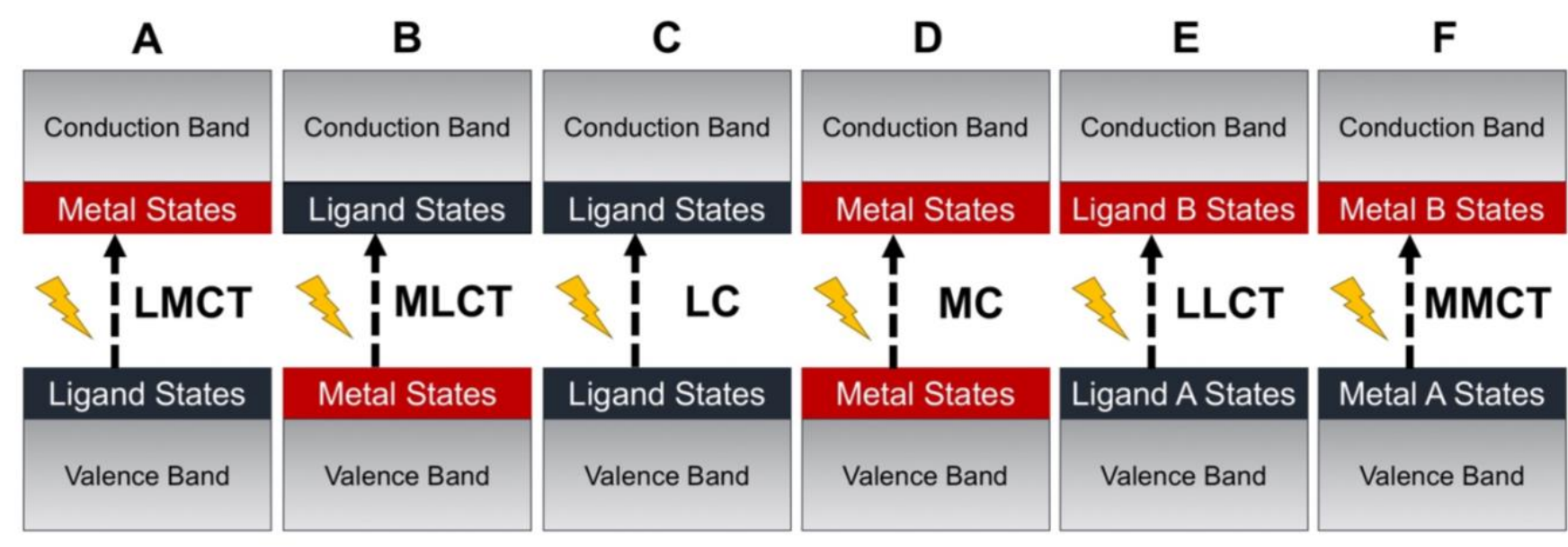

Figure 1. Representation of the six types of low-lying excited states that can be found in MOFs as a result of the different character of the Valence Band and Conduction Band edges. Photoinduced charge separation can be obtained from A ligand-to-metal charge transfer (LMCT) and B metal-to-ligand charge transfer (MLCT). Local emission can be obtained from $\mathbf{C}$ ligand-centered (LC) and $\mathbf{D}$ metal centered (MC) excited states. In bi-ligand and bi-metallic MOFs, E ligand-to-ligand charge transfer (LLCT) and $\mathbf{F}$ metal-to-metal charge transfer (MMCT) is also possible. In A-D, black and red colors represent Ligand or Metal States, respectively. In E(F), black is Ligand1(Metal1) and red Ligand2(Metal2).

Efficient charge-carriers separation relies not only on the spatial separation of the photo-generated electron-hole pairs upon excitation but also on the available charge transport pathways, i.e., photoconductivity. MOFs tend to be poor photoconductors as a result of their porosity and modular nature. The large voids usually lead to a large separation between the redox-active units in the MOF structure, which impedes the through-space interaction and thus, the (hopping) electron transport. In addition, poor organic-inorganic electronic conjugation may also hinder efficient through-bond charge-transport via delocalized conducting bands. ${ }^{24}$ Currently, increasing efforts are devoted to developing highly conductive and photoconductive MOFs, ${ }^{25,26,27}$ with conductivity values up to $10^{3} \mathrm{~S}$ $\mathrm{cm}^{-1}$. For instance, it has been shown that MOFs displaying one-dimensional (1D) Ti-O chains will facilitate electron mobility. ${ }^{28,29}$ Similarly, high electrical conductivities have been reported for MOF materials consisting of metal ions connected into stacked two-dimensional (2D) $\pi$-conjugated sheets..$^{30,31}$ The outstanding performance of these systems originates in the dispersion of their electronic band structure, ${ }^{32}$ which in turn can be directly related to the effective mass of the charge carriers. ${ }^{33}$

Elucidating the charge separation and charge carrier mobility from electronic structure calculations will not only provide insights into the fundamental properties of MOFs materials but also aid in the discovery of novel systems with improved characteristics for photocatalysis. In this context, high-throughput (HT) screening techniques become particularly useful to encompass the almost unlimited number of hypothetical structures that can be synthesized from distinct metal-node and organic-linker combinations. ${ }^{34,35,36}$ To date, tens of thousands of different experimental MOF structures have been reported and collected in extended databases. ${ }^{37,38}$ These databases provide the basis for computational studies devoted to identifying promising materials for different applications, ${ }^{39}$ as well as to produce rich data to be exploited for Machine-Learning (ML) purposes. ${ }^{40} \mathrm{HT}$ screening procedures require the development of automatized computational protocols that translate the desired physicochemical 
properties into computable descriptors, such as distances, energies or any other quantifiable property. These descriptors need to be computed accurately to be predictive but also at a sufficiently low computational cost such that they are viable for HT screenings.

In a recent paper, we proposed a computational protocol to address visible-light absorbance and band-edge energies using cost-effective Density-Functional-Theory (DFT) methods that can be applied for high-throughput screening of large databases of MOF structures. ${ }^{41}$ In this work, we extend this computational strategy to evaluate charge separation and charge-carrier mobility in MOF crystals from cost-effective DFT calculations. To do so, we selected 15 MOF structures that have been reported as promising candidates for photocatalysis. We first analyze their light absorption and photo-redox properties in terms of their optical band gap and band-edge energies. Then, we quantify light-induced charge separation by calculating the spatial overlap between the electron and hole densities of the lowest-lying singlet excited state, ${ }^{42,43,44}$ and discuss three different strategies to estimate it from ground state calculations in large MOF databases. Finally, we address the evaluation of the charge carrier mobility using the electron and hole effective masses within the band transport model. ${ }^{45}$ Altogether, our results elucidate the different electronic structure features at the basis of the most common strategies used in the design of MOFs for photocatalysis, namely, visible-light absorption, band-edge adequacy, charge separation, and electron mobility. By considering these four descriptors, we aim to provide a complete picture of the main characteristics required for efficient photocatalysis while presenting a cost-effective procedure that can be used in HT screenings to accelerate the discovery of efficient photocatalytically active MOFs.

Table 1. Photocatalytically active MOFs studied in this work, labelled as $\mathbf{1 5}$-set. Information about the metal and ligand constituent units is given, as well as its use for photocatalysis.

\begin{tabular}{|c|c|c|c|c|}
\hline MOF & Metal & Ligand & Photocatalvsis & Ref. \\
\hline AIPMOF & Al(IIII) & porphyrin & $\mathrm{H}_{2}$ production & \\
\hline MIL125 & Ti(IV) & 1.4-benzenedicarboxvlate (bdc) & - & 47 \\
\hline MIL125- & $\mathrm{Ti}(\mathrm{IV})$ & 2-amino-1,4-benzenedicarboxvlate & $\mathrm{H}_{2}$ production & 48 \\
\hline MOF- & $\mathrm{Zn}(\mathrm{II})$ & 2,5-dioxido-1,4-benzenedicarboxvlate & - & 49 \\
\hline MOF5 & $\mathrm{Zn}(\mathrm{II})$ & 1,4-benzenedicarboxvlate & propene oxidation & 50 \\
\hline MOF5- & $\mathrm{Zn}(\mathrm{II})$ & 2-amino-1,4-benzenedicarboxylate & - & 51 \\
\hline MOFZn1 & $\mathrm{Zn}(\mathrm{II})$ & 1,2,3-benzotriazole-5-carboxvlate & $\mathrm{CO}$, methanation & 52 \\
\hline MUV11 & Ti(IV) & benzene-1,4-dihvdroxamate & $\mathrm{H}_{2}$ production & 53 \\
\hline NNU36 & $\mathrm{Zn}(\mathrm{II})$ & 9,10-bis(4'-pyridylethynyl)-anthracene & $\mathrm{Cr}(\mathrm{VI})$ reduction and dye & 54 \\
\hline NTU-9 & $\mathrm{Ti}(\mathrm{IV})$ & 2,5-dihydroxvterephthalate & dve degradation & 55 \\
\hline UiO66 & $\mathrm{Zr}(\mathrm{IV})$ & 1,4-benzenedicarboxvlate & - & 56 \\
\hline UiO66- & $\mathrm{Zr}(\mathrm{IV})$ & 2-amino-1,4-benzenedicarboxvlate & $\mathrm{H}_{2}$ production & 57 \\
\hline $\mathrm{Zn}, \pi \mathrm{TFB}$ & $\mathrm{Zn}(I I)$ & tetrathiafulvalene(TTF)-tetrabenzoate & & 58 \\
\hline ZSTU-1 & Ti(IV) & 4.4', $4^{\prime \prime}-$ nitrilotribenzoate & $\mathrm{H}_{2}$ production & 29 \\
\hline ZSTU-2 & $\mathrm{Ti}(\mathrm{IV})$ & 1,3,5-tris(4-carboxyphenvl) benzene & $\mathrm{H}_{2}$ production & 29 \\
\hline
\end{tabular}

\section{Methods}

\section{MOF structure dataset}

We selected 15 MOFs structures (herein labelled 15-set) for which photocatalytic activity has been reported or that show promising features for photocatalysis. The chemical composition in terms of metal and ligand subunits, as well as the photocatalytic reaction for which they have been used, are collected in Table 1. In this dataset, we considered non-magnetic semiconducting MOFs built from $\mathrm{Al}(\mathrm{III}), \mathrm{Ti}(\mathrm{IV}), \mathrm{Zn}(\mathrm{II})$, and $\mathrm{Zr}(\mathrm{IV})$ metal centers and 11 distinct ligands. We included chemically similar systems such as MIL125 and MIL125- $\mathrm{NH}_{2}$, that share the metal and 
structural topology but differ in the $-\mathrm{NH}_{2}$ substitution of the 1,4-benzenedicarboxylate (bdc) ligand. The same applies to UiO66 and UiO66- $\mathrm{NH}_{2}$, as well as MOF5 and MOF5- $\mathrm{NH}_{2}$. At the same time, MIL125(- $\left.\mathrm{NH}_{2}\right), \mathrm{UiO} 66\left(-\mathrm{NH}_{2}\right)$ and MOF5(- $\left.\mathrm{NH}_{2}\right)$ are built from the same bdc ligand but differ in their metal being $\mathrm{Ti}(\mathrm{IV}), \mathrm{Zr}(\mathrm{IV})$, and $\mathrm{Zn}(\mathrm{II})$, respectively. The latter implies also important changes in their structural motifs. MOFZn1 and MOF-74Zn are structurally similar MOFs showing a rod-like framework of Zn(II), but they have different ligands. ZSTU-1 and ZSTU2 have also similar structural frameworks built from $\mathrm{TiO}_{2} 1 \mathrm{D}$ channels but differ in their ligands. AlPMOF was included as a representative example of a porphyrin-based MOF. MUV11 and NTU-9 are Ti(IV)-based MOFs specifically designed for photocatalysis, as well as the pillared-layer NNU36 Zn(II)-MOF, built from a photo-active bipyridine-anthracene ligand. Finally, $Z \mathrm{n}_{2}$ TTFB was reported to exhibit high charge mobilities through TTF-stacked motifs. Altogether, the different metals, ligands, and topologies of the MOFs considered here cover the main strategies that have been used in the literature to improve the photocatalytic performance of MOFs.

\section{Computational details}

Geometry optimization of the crystal coordinates and cell parameters of all MOFs was performed under periodic boundary conditions with density-functional theory (DFT) at PBE ${ }^{59}$ level including D3 dispersion correction ${ }^{60}$ using the CP2K program version 6.1. ${ }^{61}$ In all calculations we used the Goedecker-Teter-Hutter pseudopotentials ${ }^{62}$ with a density cutoff of $450 \mathrm{Ry}$ and DZVP-MOLOPT basis set for non-metal atoms and TZVP-MOLOPT basis set for $\mathrm{Al} / \mathrm{Zn} / \mathrm{Ti} / \mathrm{Zr}$ metal atoms. ${ }^{63}$ The optimized cell parameters are collected and compared to the experimental values in Table S1. They show good agreement in all cases. Energy calculations were performed upon the optimized structures at PBEO level. ${ }^{64}$ To reduce the computational cost, the Auxiliary Density Matrix Method (ADMM) $)^{65}$ was used considering the pFIT3 auxiliary basis set for non-metal atoms ( 3 Gaussian exponents per valence orbital, includes polarization d-functions), the cFIT9 for Al atoms, and the cFIT11 for Zn/Ti/Zr atoms (contracted, $4 \mathrm{~s}, 3 \mathrm{p}$, and $3 \mathrm{~d}$ shells and $1 \mathrm{f}$ shell in total).

\section{Charge Separation}

Linear-response-time-dependent-DFT (LR-TDDFT) calculations at PBEO level were performed in periodic boundary conditions using the Tamm-Dancoff approximation as implemented in CP2K to evaluate the optical gap and to assess the charge separation in the lowest excited singlet state $S_{1}$. Only the first three states were computed. The charge separation (also named charge transfer) characterizing $S_{1}$ is calculated as the weighted average of the spatial overlap between the Kohn-Sham occupied and virtual orbitals involved in the excitation as: ${ }^{42,43,44}$

$$
\Lambda=\frac{\sum_{i, a} \kappa_{i a}^{2} O_{i a}}{\sum_{i, a} \kappa_{i a}^{2}}
$$

where $\kappa_{i a}$ are the coefficients or amplitudes associated with a given occupied-virtual pair contribution and $O_{i a}$ is the spatial overlap between these occupied and virtual orbitals, which is given by the inner product of the moduli of the two orbitals as:

$$
O_{i a}=\left\langle\left|\varphi_{i}\right||| \varphi_{a} \mid\right\rangle=\int\left|\varphi_{i}(\boldsymbol{r})\right|\left|\varphi_{a}(\boldsymbol{r})\right| d \boldsymbol{r}
$$

The spatial overlap $O_{i a}$ has been also computed between the highest occupied crystal orbital (HOCO) and lowest unoccupied crystal orbital (LUCO) of the ground state singlet, triplet and charged doublets following the three schemes described in the Results.

\section{Charge Carrier Effective Mass}


To evaluate the photoconductive properties and the mobility of the charge carriers we performed band structure calculations for the 15-set structures at the PBE level in the CP2K implementation. The reciprocal space path within the first Brillouin zone (BZ) was computed connecting the high-symmetry points using the Seek-path package. ${ }^{66} \mathrm{We}$ used 50 points integrated between each high-symmetry point in the whole path. The integration of the BZ was conducted at the $\Gamma$-point. We also computed the band structure using a Monkhorst-Pack special $\Gamma$-centered k-point grid of $2 \times 2 \times 2$ to sample the BZ. The results show no difference with the $\Gamma$-point band structure calculations (Figure S1) indicating that $\Gamma$-point integration is a good approximation for materials like MOFs with large cell parameters.

The effective mass of the charge carriers, $m^{*}$, was computed at the Conduction Band Minima (CBM) and Valence Band Maxima (VBM) for electron and holes, respectively, as the inverse of the electronic dispersion in the reciprocal space:

$$
\frac{1}{m^{*}}=\frac{1}{\hbar^{2}} \frac{\partial^{2} E}{\partial k^{2}}
$$

Using this relationship, the data extracted from the band structure calculations was fitted to a parabola to obtain the effective mass values. All high-symmetry points of the band structure path were considered to compute the effective mass. Among the different values obtained, only the smallest effective mass at the CBM and VBM is discussed as the most favorable photoconductive pathway.

\section{Results}

\section{Optical and photo-redox properties}

As mentioned in the introduction, the potential of MOFs as photocatalysts is subject to their light-harvesting and photo-redox properties. These two features constitute the main characteristics that will discriminate between energetically capable and non-capable systems in large-scale screenings. By means of electronic structure calculations, the optical bandgap can be obtained from the energy of the lowest active excited state, while the photo-redox capabilities can be estimated from the band edge positions. The accuracy of the computed values will largely depend on the level of theory. For large-scale screenings, however, cost-effective strategies are needed. In a recent article, we showed that DFT calculations at the PBE level can be empirically shifted to estimate more accurate PBEO values for the accelerated screening of MOFs for photocatalysis. ${ }^{41}$

A comparison between the PBE and PBEO bandgap values of the 15-set (see MOF dataset in Methods) is shown in Figure 2A. It can be seen that PBE systematically underestimates the electronic bandgap by ca. $1.5 \mathrm{eV}$ in comparison to the PBEO values. This agrees with our previous studies ${ }^{41}$ and with the well-known problem of generalized gradient approximation (GGA) functionals to correctly predict the bandgap in semiconductors. ${ }^{67}$ In contrast, hybrid schemes such as PBEO significantly improve the prediction. ${ }^{68} \mathrm{~A}$ comparison between the experimental optical bandgap and the computed $\mathrm{S}_{1}$ energies at the PBEO level is shown in Figure 2B. The $\mathrm{S}_{1}$ energies slightly overestimate the experimentally reported values (about $0.3 \mathrm{eV}$ on average), however, the trends are properly captured. Certainly, the accurate prediction of the optical properties of MOF materials is still challenging and will largely ben efit from the development of novel methods and computational strategies. For large-scale screenings, we found that an empirical shift of $+0.85 \mathrm{eV}$ applied to the PBE values provides reasonable estimates of the lowest absorption edge of MOF materials at a very low computational $\operatorname{cost}^{41}$ (Figure 2C).

The photo-redox properties of the 15-set have been addressed by means of estimating the absolute position of the conduction (CB) and valence (VB) band edges. ${ }^{13,41}$ The capability of a MOF to promote a particular redox reaction upon the absorption of light is then given by the position of the CBM (for reduction) and the VBM (for oxidation) relative to a target redox potential. In Figure 3, the CBM and VBM computed energies are shown with respect to 
the $\mathrm{H}^{+} / \mathrm{H}_{2}$ reduction and $\mathrm{H}_{2} \mathrm{O} / \mathrm{O}_{2}$ oxidation potentials. In all cases, the $\mathrm{CBM}$ is placed above the $\mathrm{H}^{+} / \mathrm{H}_{2}$ indicating thermodynamic adequacy for hydrogen production. In contrast, some cases display VB edges above $\mathrm{H}_{2} \mathrm{O} / \mathrm{O}_{2}$, which will prevent the oxidation of water and thus necessitates the use of a sacrificial agent. It is worth mentioning that only half of the 15-set systems (AIPMOF, MIL125(-NH $)$, MUV11, UiO66(- $\left.\mathrm{NH}_{2}\right)$ and ZSTU-) were originally targeted for $\mathrm{H}^{+} / \mathrm{H}_{2}$ reaction, while the others have been used for different photocatalytic reactions such as $\mathrm{CO}_{2}$ reduction or organic degradation (Table 1). In this context, the band edges can be compared with the redox potential of the reactions of interest when performing computational screening of MOFs for photocatalysis. In principle, this option opens the possibility to avoid certain reactions and thus, promote selectivity in competitive photochemical processes. ${ }^{69}$ Altogether, our computational strategy allows to easily estimate optical absorption and band energy alignment of MOF structures, which in turn enables to focus the experimental effort only towards the promising systems.
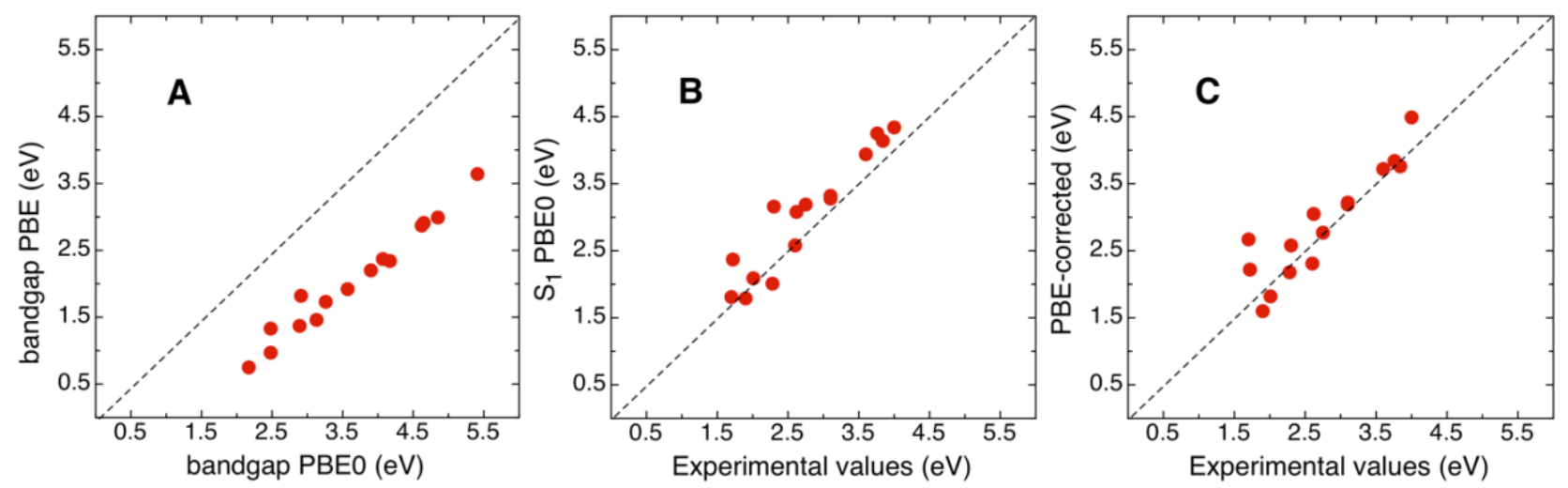

Figure 2. Comparison between $A$ : the PBE and PBEO bandgaps, $B$ : the experimental values and the $\mathrm{S}_{1}(\mathrm{PBE})$ energies and $\mathbf{C}$ : the experimental values and the corrected PBE energies $(+0.85 \mathrm{eV})^{41}$ of the 15 -set MOFs. Raw data is given in Table S2.

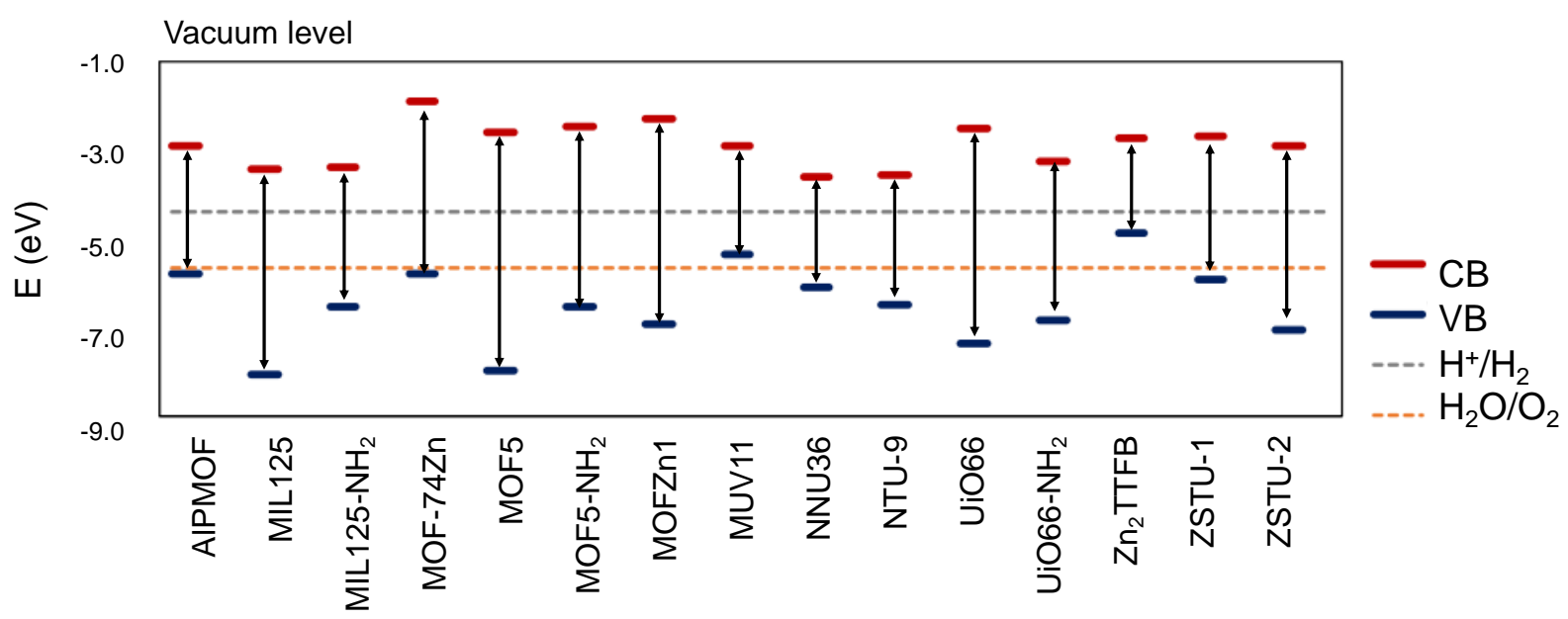


Figure 3. Conduction band ( $\mathrm{CB}$ ) and valence band (VB) edge energies computed with PBEO for the $\mathbf{1 5}$-set MOFs. The $\mathrm{H}^{+} / \mathrm{H}_{2}$ and $\mathrm{H}_{2} \mathrm{O} / \mathrm{O}_{2}$ potentials are indicated. Raw data is given in Table $\mathbf{S 2}$.

\section{Charge separation}

Photo-generated charge separation is one of the crucial steps controlling the conversion efficiency in photocatalysis. A common scenario of a MOF photocatalytic system includes an electron-withdrawing co-catalyst and an easily oxidizable sacrificial agent. ${ }^{70}$ In this way, the MOF acts as the photosensitizer and the electron-hole recombination is prevented by favoring charge transfer to the external components. However, the charge carrier lifetime needs to be long enough within the MOF to maximize the chances of electron(hole) injection at the interfaces or to be directly transferred to the substrates. In this context, the formation of long-lived charge-transfer excited states in MOFs is a promising design target that can be exploited for large-scale computational screenings. To evaluate the charge transfer (CT) character of an excited state, one possibility is to quantify the charge separation $\Lambda$ as the spatial overlap between the photo-generated electron-hole pair densities (see Methods). We assume that long-lived electron-hole pairs will decay to the lowest excited $S_{1}$ state. ${ }^{71}$ The $\Lambda$ values that characterized the $S_{1}$ state of the 15-set are collected in Table 2. They range from 0.11 to 0.76 and have been computed considering all relevant one-electron transitions involved in the $S_{1}$ state (see Methods). Small $\Lambda\left(S_{1}\right)$ values indicate that the electron and hole densities do not spatially overlap and thus that $S_{1}$ is mainly a CT state. In contrast, large $\Lambda\left(S_{1}\right)$ values are associated with local excitations that can be either ligand- or metal-centered. Remarkably, the results show that the $\mathrm{CT}$ or local character of $\mathrm{S}_{1}$ cannot be unequivocally assigned as 0 or 1 , but that most of the systems show a partial character of both.

Table 2. Charge separation coefficient $\Lambda$ characterizing $S_{1}$ state obtained at PBEO, and charge carrier effective mass at the VBM (hole) and CBM (electron) using PBE, in electron rest mass $\left(m_{0}\right)$ units.

\begin{tabular}{|l|l|l|l|}
\hline & $\Lambda\left(\mathrm{S}_{1}\right)$ & $m^{*}{ }_{\text {hole }}$ & $m^{{ }_{\text {electron }}}$ \\
\hline AlPMOF & 0.70 & 30.0 & 30.1 \\
\hline MIL125 & 0.33 & - & 0.9 \\
\hline MIL125-NH 2 & 0.24 & 84.2 & 4.2 \\
\hline MOF-74Zn & 0.56 & 7.3 & 5.2 \\
\hline MOF5 & 0.40 & - & 217.1 \\
\hline MOF5-NH 2 & 0.32 & 62117.8 & 67.4 \\
\hline MOFZn1 & 0.64 & - & 3.3 \\
\hline MUV11 & 0.25 & 12.8 & 12.5 \\
\hline NNU36 & 0.76 & 1.2 & 3.7 \\
\hline NTU-9 & 0.58 & 1.1 & 1.6 \\
\hline UiO66 & 0.59 & - & 0.8 \\
\hline UiO66-NH 2 & 0.39 & 104.9 & 0.8 \\
\hline Zn2TTFB & 0.38 & 2.2 & 16.8 \\
\hline ZSTU-1 & 0.11 & 7.0 & 8.9 \\
\hline ZSTU-2 & 0.30 & - & 16.8 \\
\hline
\end{tabular}

Interestingly, chemically and structurally similar MOFs such as MIL125 and MIL125- $\mathrm{NH}_{2}$ present non-equivalent $\Lambda\left(\mathrm{S}_{1}\right)$ values, being slightly smaller for the $-\mathrm{NH}_{2}$ substituted one. This is also the case for MOF5 and MOF5-NH $\mathrm{N}_{2}$ as well as for UiO66 and UiO66- $\mathrm{NH}_{2}$ thus clearly indicating the participation of the $-\mathrm{NH}_{2}$ groups in the low lying states. ${ }^{12,15}$ Comparison between the Ti(IV)-, $\mathrm{Zn}$ (II)- and Zr(IV)-based MOFs MIL125(- $\left.\mathrm{NH}_{2}\right)$, MOF5(-NH 2 ), and UiO66($\left.\mathrm{NH}_{2}\right)$, respectively, points also to the role of the metal in tuning the CT capabilities. Lower $\Lambda\left(\mathrm{S}_{1}\right)$ values are obtained for the Ti(IV)-MIL125 MOF followed by the Zn(II)-MOF5 and the Zr(IV)-UiO66 cases. The predicted ability of MIL125$\mathrm{NH}_{2}$ to undergo efficient photo-induced charge separation agrees with previously reported experimental evidence from transient absorption spectroscopy. ${ }^{72}$ Small $\Lambda\left(S_{1}\right)$ values are also obtained for the Ti(IV)- ZSTU- systems, for 
which charge separation was predicted based on their chemical composition and 1D structural motif. ${ }^{29}$ In particular, ZSTU-1 showed a better photocatalytic performance than ZSTU-2 which could be in part due to a more favorable charge separation, as predicted by our $\Lambda\left(\mathrm{S}_{1}\right)$ approach. From our results, we can conclude that both the ligand and the metal ion can be tuned to modify the CT character of the lowest state. Particularly promising are MOFs such as MIL125-NH2, MUV11 and ZSTU-1 that combine Ti(IV) with oxidizable ligands leading to low-lying LMCT (Figure 1A).

Being able to quantify the photo-induced charge separation in MOFs is certainly a step forward into the characterization of their optical properties from electronic structure calculations, which was so far limited to the interpretation of the $C B$ and $V B$ character. Evaluating $\Lambda\left(\mathrm{S}_{1}\right)$, however, implies the calculation of the lowest excited state $S_{1}$ and the spatial overlap between all the occupied-unoccupied orbital pairs that characterize it. This entails a large computational cost that cannot be afforded for HT screenings. For this reason, an alternative procedure to estimate $\Lambda$ for many MOF structures needs to be established. To do so, we analyze three possible approximations to determine the electron-hole separation based on ground state (GS) calculations. One possibility is to consider the spatial overlap $O_{i a}$ between the highest occupied crystal orbital (HOCO) and lowest occupied crystal orbital (LUCO) of the GS singlet state assuming that the lowest excitation will take place between the band-edge orbitals (Figure 4A). Another possibility is to evaluate the spatial overlap between the $\operatorname{HOCO}(\alpha)$ and $\operatorname{LUCO}(\beta)$ of the lowest GS triplet (Figure 4B). This will assume that the lowest excited singlet $S_{1}$ and triplet $T_{1}$ states are equivalent. Finally, it is possible to consider the electron-hole pair as independent charges that coexist in a neutral state, therefore neglecting their interaction. In that case, the spatial overlap is evaluated between the $\operatorname{HOCO}(\alpha)$ of the electron injection (Figure 4C) and the LUCO $(\beta)$ of the hole injection (Figure 4D) both in their GS doublet. We have labelled the three strategies as GS-S, GS-T, and GS-D, respectively.

A

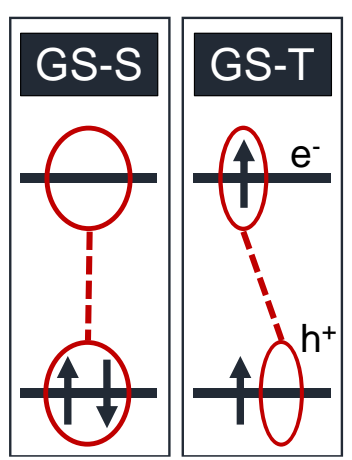

C

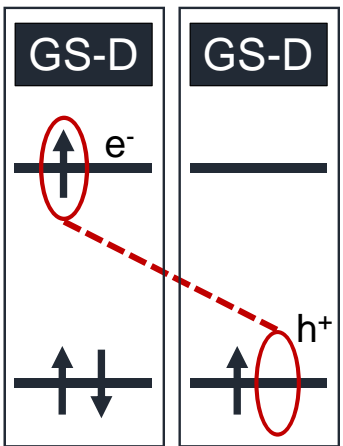

Figure 4. Estimations considered to evaluate the $C T$ character of $\mathrm{S}_{1}$ from ground state calculations: $A$ ground state singlet (GS-S), the spatial overlap is evaluated between the HOCO and the LUCO; B GS triplet (GS-T), the spatial overlap is computed between the $\mathrm{HOCO}(\alpha)$ and $\operatorname{LUCO}(\beta)$; C GS doublet (GS-D) when injecting an electron (e $\mathrm{e}^{-}$) and D GS doublet when injecting a hole $\left(\mathrm{h}^{+}\right)$, the spatial overlap is evaluated between the $\operatorname{HOCO}(\alpha)$ of the $\mathrm{e}^{-}$injection and the $L U C O(\beta)$ of $h^{+}$injection. 


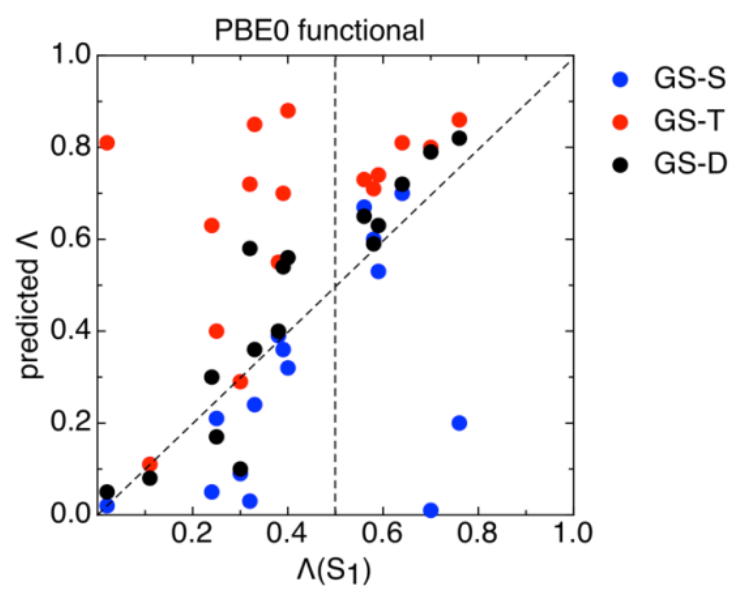

Figure 5. Comparison between the computed $\Lambda\left(\mathrm{S}_{1}\right)$ and the predicted $\Lambda$ values obtained by means of the GS-S, GST or GS-D protocol, as explained in the main text. Raw data is given in Table S3.
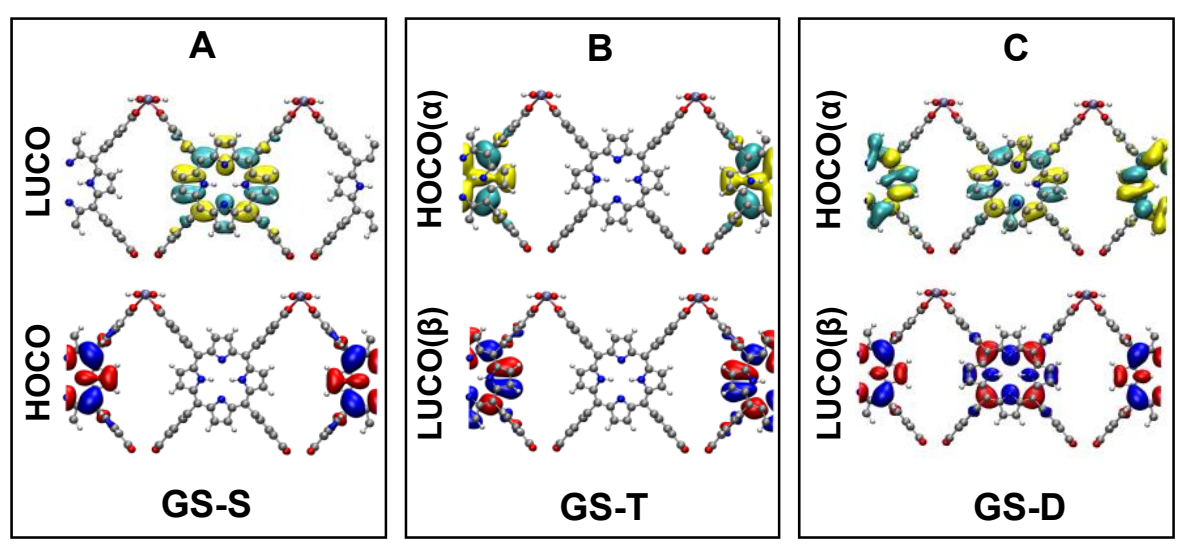

Figure 6. HOCO and LUCO orbitals of AIPMOF computed at PBEO level following the A GS-S, B GS-T and C GS-D protocols as explained in the main text.
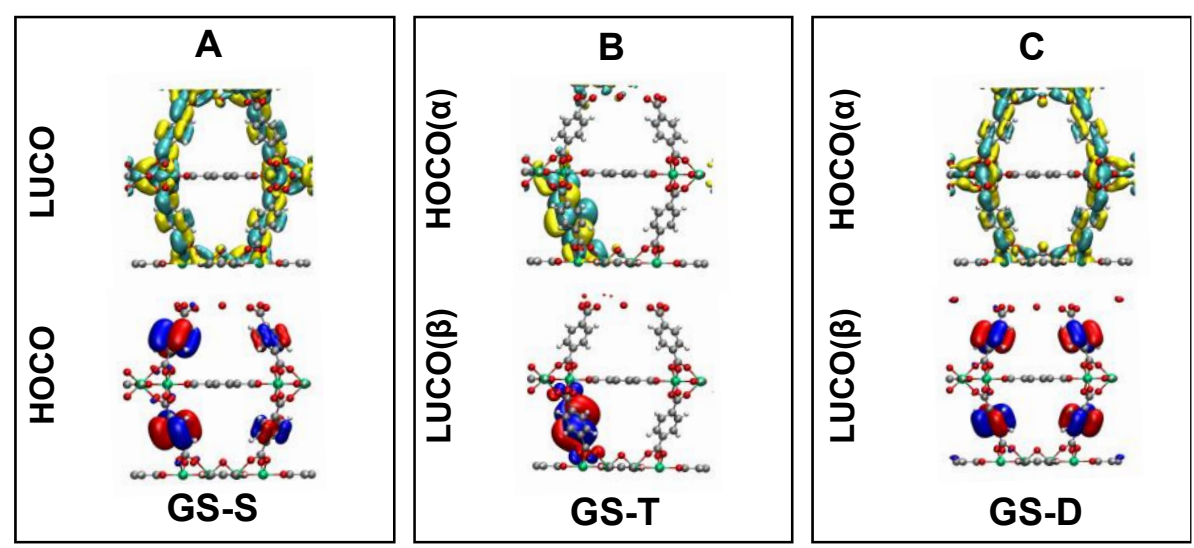

Figure 7. HOCO and LUCO orbitals of MIL125 computed at PBEO level following the A GS-S, B GS-T and C GS-D protocols as explained in the main text.

Comparison between the computed $\Lambda\left(S_{1}\right)$ and the values obtained by means of the three aforementioned strategies (predicted $\Lambda$ ) is shown in Figure $\mathbf{5}$. The GS-S protocol predicts very small $\Lambda$ in cases with large $\Lambda\left(\mathrm{S}_{1}\right)$ reference values, that is, it will erroneously predict low-lying CT excitations for systems characterized by a mainly local $\mathrm{S}_{1}$ state. In 
contrast, the GS-T protocol overestimates $\Lambda$ with respect to the $\Lambda\left(\mathrm{S}_{1}\right)$ reference values in many cases, thus it would automatically discard promising CT candidates throughout screening. Finally, the GS-D strategy reproduces correctly the $\Lambda\left(\mathrm{S}_{1}\right)$ trend with minor deviations in most cases, that is, considering the reduced and oxidized MOF in its GS we can estimate the locality of the e- and the $\mathrm{h}+$ in $\mathrm{S} 1$, respectively. In order to understand the different behavior of the GS-S, GS-T, and GS-D protocols we analyze the results of two test-cases in the following.

We have selected AIPMOF as a representative MOF displaying a low-lying local excitation with an $\Lambda\left(\mathrm{S}_{1}\right)$ value of 0.70 . This excitation corresponds to a $\pi-\pi^{*}$ transition from the highest occupied molecular orbital (HOMO) to the lowest unoccupied molecular orbital (LUMO) of the porphyrin ligand, also known as the $Q$ bands. ${ }^{46}$ The local character of the lowest state is well captured by the GS-T and GS-D strategies, which predict $\Lambda$ values of 0.80 and 0.79 , respectively. However, the GS-S protocol misleads this value to 0.01 . These results can be explained by analyzing the different HOCO and LUCO obtained in each case (Figure 6). First, one has to notice that the unit cell contains four porphyrin units organized in two columns following eclipsed $\pi$-stacking motifs. In the GS-S, the crystal orbitals are delocalized along one or another column of the unit cell and therefore, the spatial overlap between them is negligible (Figure 6A). In contrast, the frontier crystal orbitals are both localized in a single porphyrin unit when evaluating the GS-T and thus, result in a large $\Lambda$ (Figure 6B). Finally, delocalized orbitals in the four porphyrins are shown for the electron and hole injection states in GS-D, which lead also to a large $\Lambda$ (Figure $6 \mathrm{C}$ ). The different (de)localization of the crystal orbitals in each approximation determines its ability to predict $\Lambda$ and originates in the different electronic features that characterize the ground state singlet, triplet, and charged doublet states. On the one hand, the two spatially separated $\pi$-stacking motifs of the unit cell result in pseudo-degenerate HOCOs (and LUCOs) in the GS-S that would erroneously predict CT from one to another. On the other hand, both the GS-T and GS-D strategies will predict low-lying local excitations, but for very different reasons: while GS-T localizes the frontier crystal orbitals in the same porphyrin unit, in GS-D the injected charges delocalize them in the four porphyrin units of the unit cell.

We applied the same analysis for MIL125 as an example of MOF characterized by a low-lying CT excitation with an $\Lambda\left(\mathrm{S}_{1}\right)$ value of 0.33 . The latter involves an electronic transition from the 1,4-benzenedicarboxylate (bdc) ligand to a hybrid ligand/Ti-cluster band. ${ }^{12}$ In this case, both the GS-S and GS-D strategies predict a CT character for $\mathrm{S}_{1}$ with an estimated $\Lambda$ of 0.24 and 0.36 , respectively, whereas GS-T will consider that the lowest state is local with an estimated $\Lambda$ of 0.85 . The frontier crystal orbitals obtained in each case are shown in Figure 7. Both GS-S and GS-D strategies represent correctly the different nature of the valence and conduction band edges. However, the GS-T minimizes its energy through fully localizing the frontier orbitals in one ligand unit. This example illustrates that the lowest triplet is not electronically equivalent to the lowest excited singlet, especially when local excitations and CT compete. This is because electronic exchange interactions play a crucial role in stabilizing local excitations and lead to large singlet-triplet energy splitting. ${ }^{73}$ This conclusion would be of key importance when considering electrogenerated excited states instead of light-induced excitations. In the former case, the statistical ratio of triplet and singlet excitons induced by the applied voltage is 3:1, while in the latter only singlet excitations are accessible in the absence of spin-orbit coupling (SOC) effects. ${ }^{74}$

Our analysis on the AIPMOF and MIL125 examples illustrates that (i) local excitations may not be well represented by the HOCO and LUCO of the GS-S due to orbital pseudo-degeneracies, (ii) the GS-T might not be representative of the lowest excited singlet if the latter is characterized by a strong CT character and (iii) the independent injection of an electron and hole in their GS-D can be used to evaluate charge separation in $S_{1}$ and to discriminate between mainly local $(\Lambda>0.5)$ and mainly $\mathrm{CT}(\Lambda<0.5)$ excitations. The conclusions (i) and (iii) equally apply when estimating $\Lambda\left(S_{1}\right)$ from ground state calculations performed with PBE functional, which is the preferred functional for HT screening. ${ }^{41}$ Interestingly, the GS-T predictions when using PBE nicely follow the trend of the GS-D results and do 
not significantly deviate from the $\Lambda\left(\mathrm{S}_{1}\right)$ reference values, as was shown by the PBE0 results (Figure S2). This is a direct consequence of the lack of exact exchange in GGA functionals and, although they may misassign the correct character of the triplet state, they are useful for the evaluation of the charge separation propensity of $S_{1}$ in large MOF databases via cost-effective ground state calculations.

\section{Charge mobility}

In the band transport model, the mobility of the charge carriers is associated with the dispersion of the CB minimum and VB maximum. This is usually small in MOFs, for which most charge-transport studies reveal a hopping transport regime. ${ }^{75,76}$ In the latter, the charge carriers are localized on discrete sites of the MOFs and move from one site to another by means of thermally activated jumps. Generally, it is assumed that if the band-transport model predicts low mobility, that would be also the case when considering hopping. ${ }^{45}$ That is, in the limit of the validity of the band transport mechanism i.e., when the band dispersion is zero, the hopping regime will also lead to small mobility values. Hence, we use herein the band transport model to evaluate the electron and hole mobilities in terms of their effective mass $m^{*}$. The effective mass is calculated directly from the band structure (see Methods) and provides a measure of the photoconductive capabilities. Because $m^{*}$ is inversely proportional to the charge mobility, large/small values of $m^{*}$ will be associated with poor/high photoconductive performance.

Band structure calculations were performed for the 15-set MOF structures at the PBE level (Figure S3). To ensure that PBE functional provides reliable VBM and CBM bands, we compared the Projected Density of States (PDOS) of each MOF computed with PBE and PBE0. The results are plotted in Figure S4 and show that in most cases the only difference is a down-shift of the energy of the CBM that results in a smaller bandgap when using PBE as compared with PBE0. In those cases, both methods will provide a similar band dispersion at the VBM and CBM and thus, costeffective PBE calculations are appropriate. In contrast, a drastic change in the nature of the VBM or CBM may affect the band dispersion and therefore, the estimated effective mass. This is the case of MIL125, MOF5, MOFZn1, UiO66, and ZSTU-2, for which the character of the VBM becomes significantly modified by changing the functional. For those MOFs, the addition of Hartree-Fock exchange within DFT causes a change in the contribution of the density in the valence band. The calculations with PBE showed contributions from the transition metal and oxygen atoms near the VBM, while when using PBEO those contributions are reduced and shifted to lower energies. This disagreement affects the description of the VBM in 5 of the 15 studied cases, which corresponds to $17 \%$ of the effective mass computations. For this reason, the PBE approximation to the band structure needs to be perform with caution.

The smallest $m^{*}$ computed at the VBM and CBM of the 15-set are collected in Table 2 given for the electron rest mass $m_{0}$, i.e. $\mathrm{m}^{*} / \mathrm{m}_{0}$. The values obtained range from 0.8 to $6 \times 10^{4}$ thus encompass significantly different photoconductive capabilities. For instance, the electron effective masses obtained for Al-PMOF $\left(30.1 m_{0}\right)$ and MIL$125\left(0.9 m_{0}\right)$ reflect the difference in their conduction band edge dispersion (Figure S3). While Al-PMOF shows a localized porphyrin-based LUCO (Figure 6A), MIL125 presents a ligand/Ti-cluster delocalized LUCO (Figure 7A) that is translated into a more pronounced curvature of the CBM. Efficient electron mobility is also predicted for NNU36, NTU-9, and UiO66 $\left(-\mathrm{NH}_{2}\right)$ materials, which all display a considerable dispersion in the CBM (Figure S3). Interestingly, the addition of functional groups such as $-\mathrm{NH}_{2}$ in materials like MIL125, MOF5, and UiO66 increases the hole effective mass up to $6 \cdot 10^{4} \mathrm{~m}_{0}$. The $-\mathrm{NH}_{2}$ substitution in MOFs is performed to improve the optical absorption properties, ${ }^{12}$ however, the localized nature of the $-\mathrm{NH}_{2}$ states results in flat bands at the VBM that may decrease their potential photoconductivity of holes (Table 2). Altogether, an appropriate electronic mixing of the organic and inorganic contributions in the VBM/CBM is desired to maximize charge mobilities. This metal-ligand orbital mixing can be favored for instance by promoting extended high-dimensional networks in the MOF topology, such as onedimensional or two-dimensional metal-oxygen motifs. 
a)

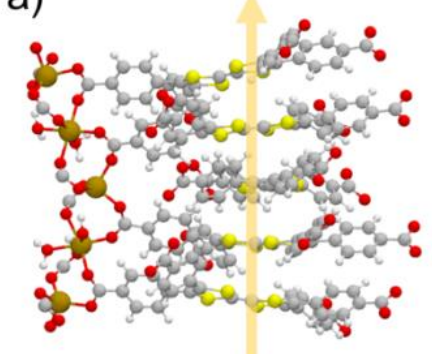

$\mathrm{Zn}_{2}$ TTFB b)

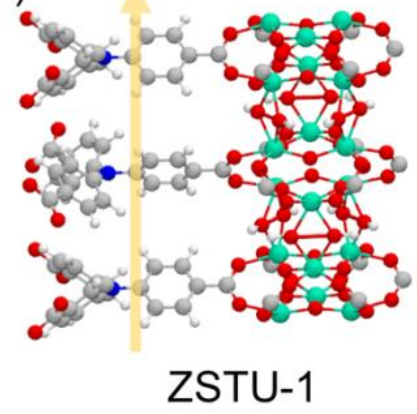

c)

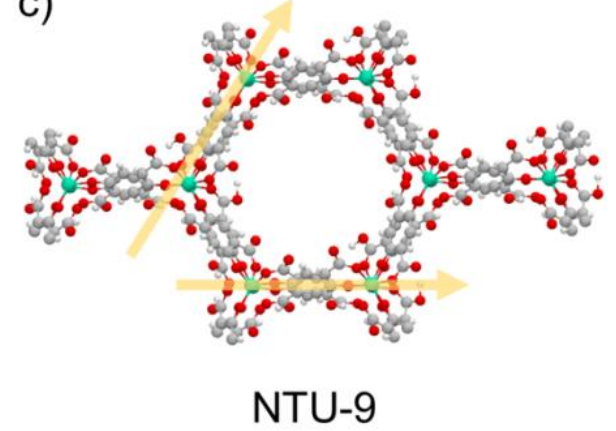

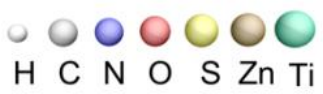

Figure 8. Representation of the computed photoconductive pathways of the lightest hole carriers in a) $Z n_{2} T T F B$, along the benzoate-extended TTF stack, b) ZSTU-1, through the ligand staking direction and c) NTU-9, along the honeycomb-like 2-dimensional layers of 2,5-dihydroxyterephthalate ligands.

An important question when addressing charge carriers mobilities is the spatial orientation of the most efficient photoconductive pathway. This information can be obtained from experimental measurements when a particular direction presents higher mobility than others. In our calculations, we obtain this information from the k-point path associated with the lowest effective mass (Table S4). Figure 8 shows the direction in real space associated with the lowest hole effective mass computed for three representative cases: $Z_{2}$ TTFB, ZSTU-1, and NTU-9. In the case of $\mathrm{Zn}_{2} \mathrm{TTFB}$, we found that the smallest hole effective mass $\left(2.2 m_{0}\right)$ is ascribed to the $Y \rightarrow \boldsymbol{\Gamma}$ path (Table S4), which in real space corresponds to the tetrathiafulvalene 1D-channel (Figure 8a). This agrees with the previously reported experimental evidence, which showed high intrinsic mobilities in $Z n_{2}$ TTFB along the helical $\pi$-stacking motif of TTF. ${ }^{58,77}$ This is also the case in ZSTU-1, for which the preferred direction of photoconductivity corresponds to the 1D-rod of $\pi$-stacked ligands (Figure 8b). ${ }^{29}$ Certainly, the presence of 1D-channels in layered MOFs may facilitate the charge transport and is a promising strategy for the design of highly conductive materials. ${ }^{31}$ However, other topologies such as two-dimensional (2D)-like systems can also exhibit low effective masses when the through-bond hybridization is optimal. This is the case of NTU-9 material, which displays a 2D- hexagonal topology with high charge mobility along with the three equivalent ligand-to-Ti(IV) directions (Figure 8c). The strong bonding between the $\mathrm{Ti}(\mathrm{IV})$ and $\mathrm{O}$ atoms enables the band dispersion along the 2D plane, while remains minor in the stacking direction. ${ }^{55}$ The characterization of the main photoconductive pathways in 3D MOFs is thus crucial to establish specific design strategies towards 1D or 2D topologies. In this context, our cost-effective computational strategy based on the effective mass approximation can capture these two structural features that promote photoconductivity. Thus, it can be applied to large-scale screening not only to identify potential photoconductive MOFs but also to characterize their charge transport motifs.

\section{Conclusions}

The inherent tunability of MOFs makes them particularly attractive for the design of high-performance photocatalysts. In this context, increasing efforts are devoted to the design of new materials with long-lived charge separation and exceptional charge carriers mobilities. These characteristics are by no means easily predictable and thus computational support is crucial. In this work, we propose two computational strategies, namely the analysis of charge separation and charge mobility, to easily address these properties in MOF structures. Based on cost- 
effective DFT calculations at the PBE level, our protocol can be applied to large databases of MOF structures and serve as a fast and comprehensive step that guide the experimental synthesis towards the most promising systems. On the one hand, our results show that the charge separation that characterizes the low-lying singlet excited state can be estimated from the spatial overlap between the $\operatorname{HOCO}(\alpha)$ and $\operatorname{LUCO}(\beta)$ of the ground state doublet of the electron and hole injection states, respectively. This approximation can be further extended to the $\mathrm{HOCO}(\alpha)$ $\operatorname{LUCO}(\beta)$ spatial overlap of the ground state triplet state when using local GGA PBE functional. On the other hand, charge carriers mobilities of MOFs can be associated with the effective mass $m^{*}$ computed from the band structure at the VBM and CBM. The later strategy provides quantitative values to $m^{*}$ and elucidates the most favorable photoconductive pathway for charge transport. This cost-effective strategy can correctly predict the charge separation and the effective mass of 15 representative MOF structures promising for photocatalysis in agreement with the literature. From our analysis, we show that those systems combining low-lying unoccupied metal states, light-harvesting ligands and extended metal-oxygen networks display all the optoelectronic characteristics to be efficient photocatalysts.

\section{Conflicts of interest}

There are no conflicts to declare.

\section{Acknowledgements}

This work has been supported by the National Centre of Competence in Research (NCCR) Materials Revolution: Computational Design and Discovery of Novel Materials (MARVEL) of the Swiss National Science Foundation (SNSF), by the SNSF Project Funding (200021_172759), and the European Research Council (ERC) under the European Union's Horizon 2020 research and innovation programme (grant agreement 666983, MaGic). Calculations were performed at the EPFL clusters and at the Swiss National Supercomputing Centre (CSCS) under project ID s1005. All the dataset files will be available on the Materials Cloud Archive at https://archive.materialscloud.org/.

\section{Notes and references}

${ }^{1}$ H-C. Zhou, J. R. Long, O. M. Yaghi, Chem. Rev. 2012, 112, 673-674.

${ }^{2}$ H.-C. Zhou, S. Kitagawa, Chem. Soc. Rev. 2014, 43, 5415-5418.

${ }^{3}$ H. Furukawa, K. E. Cordova, M. O’Keeffe, O. M. Yaghi, Science 2013, 341, 1230444.

${ }^{4}$ H. Li, K. Wang, Y. Sun, C. T. Lollar, J. Li, H.-C. Zhou, Materials Today, 2018, 21, 108-121.

${ }^{5}$ A. H. Chughtai, N. Ahmad, H. A. Younus, A. Laypkovc, F. Verpoort, Chem. Soc. Rev., 2015, 44, 6804-6849.

${ }^{6}$ S. Carrasco, Biosensors 2018, 8, 92.

${ }^{7}$ L. Lu, B. Wu, W. Shi, P. Cheng, Inorg. Chem. Front. 2019, 6, 3456-3467.

${ }^{8}$ Y. Chen, D. Wang, X. Deng, Z. Li, Catal. Sci. Technol., 2017, 7, 4893-4904.

${ }^{9}$ P. Zhang, Q. Wang, Y. Fang, W. Chen, A. A. Kirchon, M. Baci, M. Feng, V. K. Sharma, H.-C. Zhou, Metal-Organic Frameworks (MOFs) for Environmental Applications. 2019, 203-229.

${ }^{10}$ L. Alaertsa, F. Thibault-Starzyk, E. Séguin, J. F. M. Denayer, P. A. Jacobs, D. E. De Vos, Stud. Surf. Sci. Catal. 2007, 170, 1996-2003.

${ }^{11}$ G. Lan, Y-Y. Zhu, S. S. Veroneau, Z. Xu, D. Micheroni, W. Lin, J. Am. Chem. Soc. 2018, 140, 5326-5329.

${ }^{12}$ C. H. Hendon, D. Tiana, M. Fontecave, C. Sanchez, L. D'arras, C. Sassoye, L. Rozes, C. Mellot-Draznieks, A. Walsh, J. Am. Chem. Soc. 2013, 135, $10942-10945$

${ }^{13}$ K. T. Butler, C. H. Hendon, A. Walsh J. Am. Chem. Soc. 2014, 136, 2703-2706.

${ }^{14}$ S.-N. Zhao, G. Wang, D. Poelman, P. Van Der Voort, Molecules 2018, 23, 2947.

${ }^{15}$ G. Capano, F. Ambrosio, S. Kampouri, K. C. Stylianou, A. Pasquarello, B. Smit, J. Phys. Chem. C 2020, 124, 4065-4072.

${ }^{16}$ X.-P. Wu, L. Gagliardi, D. G. Truhlar, J. Am. Chem. Soc. 2018, 140, 7904-7912.

${ }^{17}$ M. A. Syzgantseva,; C. P. Ireland, F. M. Ebrahim, B. Smit, J. Am. Chem. Soc., 2019, 141, 6271-6278

${ }^{18}$ J. Zou, Q. Peng, Z. Wen, G. Zeng, Q. Xing, G. -C. Guo, Cryst. Growth Des. 2010, 10, 2613-2619.

${ }^{19}$ R.-W. Huang, Y.-S. Wei, X.-Y. Dong, X.-H. Wu, C.-X. Du, S.-Q. Zang, T. C. W. Mak Nature Chem 2017, 9, 689-697.

${ }^{20}$ J. J.Perry IV, P. L. Feng, S. T. Meek, K. Leong, F. P. Doty, M. D. Allendorf J. Mater. Chem., 2012, 22, 10235-10248.

${ }^{21}$ M. D. Allendorf, C. A. Bauer, R. K. Bhaktaa, R. J. T. Houk Chem. Soc. Rev., 2009, 38, 1330-1352.

${ }^{22}$ X.-D. Yang, R. Zhu, L. Sun, R.-Y.Guo, J. Zhang, Inorg. Chem. 2018, 57, 2724-2729.

${ }^{23}$ T. Liu, Y.-J. Zhang, S. Kanegawa, O. Sato, Angew. Chem. Int. Ed. 2010, 49, 8645 -8648.

${ }^{24}$ A. Saeki, Y. Koizumi, T. Aida, S. Seki, Acc. Chem. Res. 2012, 45, 1193-1202.

${ }^{25}$ G. Givaja, P. Amo-Ochoa, C. G. Gomez-Garcia, F. Zamora, Chem. Soc. Rev. 2012, 41, 115 -147.

${ }^{26}$ L. Sun, M. G. Campbell, M. Dinca, Angew. Chem., Int. Ed. 2016, 55, 3566-3579.

${ }^{27}$ C. H. Hendon, D. Tiana, A. Walsh, Phys. Chem. Chem. Phys. 2012, 14, 13120-13132.

${ }^{28}$ S. Wang, T. Kitao, N. Guillou, M. Wahiduzzaman, C. Martineau-Corcos, F. Nouar, A. Tissot, L. Binet, N. Ramsahye, S. Devautour-Vinot, S. Kitagawa, S. Seki, Y. Tsutsui, V. Briois, N. Steunou, G. Maurin, T. Uemura, C. Serre. Nat Commun 2018, 9, 1660.

${ }^{29}$ C. Li, H. Xu, J. Gao, W. Du, L. Shangguan, X. Zhang, R.-B. Lin, H. Wu, W. Zhou, X. Liu, J. Yao B. Chen, J. Mater. Chem. A, 2019, 7, 11928-11933.

${ }^{30}$ D. Sheberla, L. Sun, M. A. Blood-Forsythe, S. Er, C. R. Wade, C. K. Brozek, A. Aspuru-Guzik, M. Dinca, J. Am. Chem. Soc. 2014, 136, 8859-8862. 


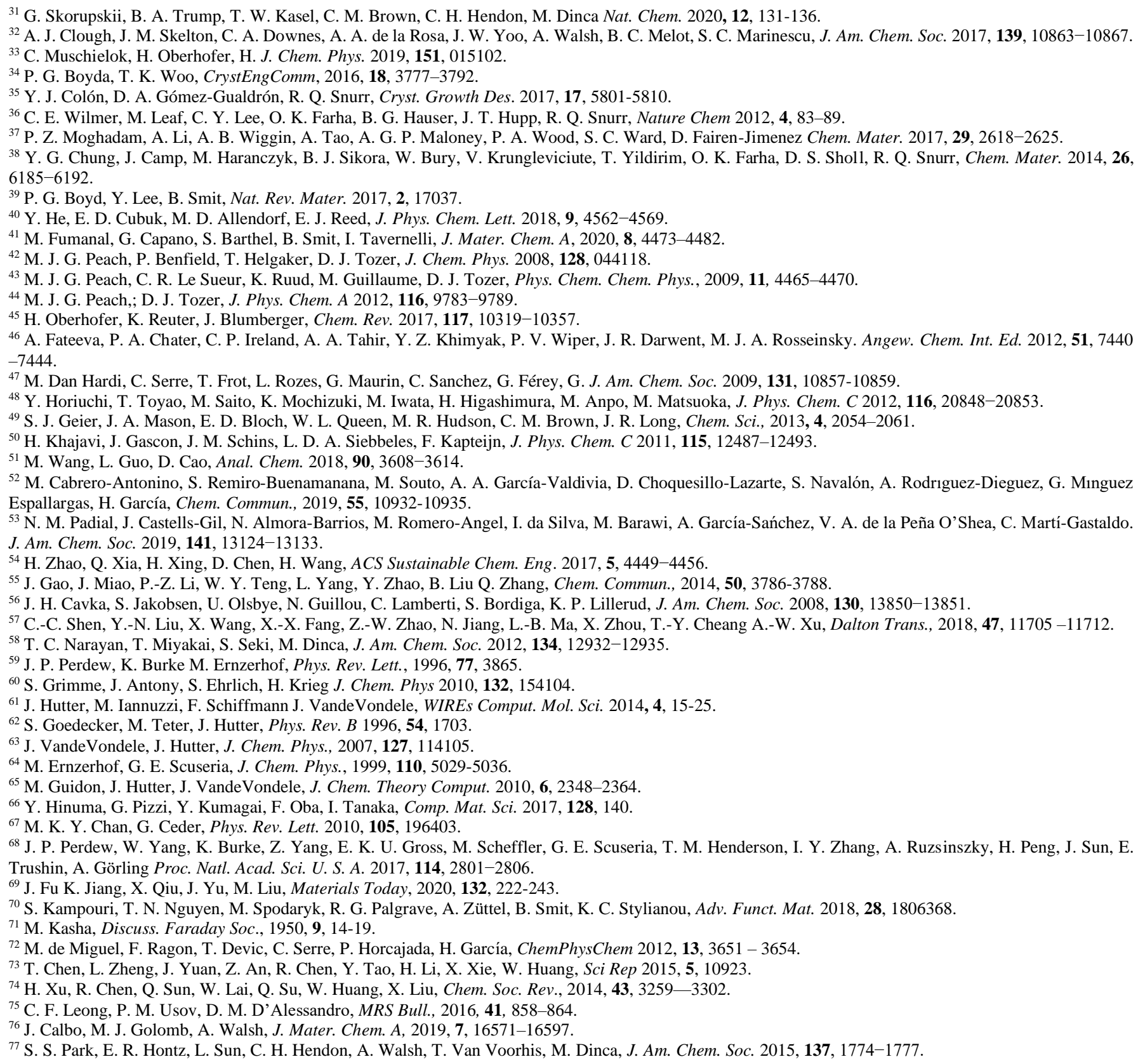

